\title{
INITIAL TESTS AND ACCURACY ASSESMENT OF A COMPACT MOBILE LASER SCANNING SYSTEM
}

\author{
K. Julge ${ }^{\text {a, } * \text {, A.Ellmann }}{ }^{\text {a }}$ T. Vajakas ${ }^{\text {b,c }}$, R. Kolka ${ }^{b}$ \\ a Tallinn University of Technology, Faculty of Civil Engineering, Department of Road Engineering, Chair of Geodesy, Ehitajate tee \\ 5, Tallinn, Estonia, 19086 - (kalev.julge, artu.ellmann)@ttu.ee \\ b Reach-U AS, Riia 24, Tartu, Estonia - (toivo.vajakas, riivo.kolka)@ reach-u.com \\ c University of Tartu, Institute of Computer Science, Liivi 2, Tartu, Estonia - tvajakas@ut.ee
}

Commission I, ICWG I/Va

KEY WORDS: Mobile Laser Scanning, LIDAR, Point Cloud, Accuracy Assessment

\begin{abstract}
:
Mobile laser scanning (MLS) is a faster and cost-effective alternative to static laser scanning, even though there is a slight trade-off in accuracy. This contribution describes a compact mobile laser scanning system mounted on a vehicle. The technical parameters of the used system components, i.e. a small LIDAR sensor Velodyne VLP-16 and a dual antenna GNSS/INS system Advanced Navigation Spatial Dual, are reviewed, along with the integration of these components for spatial data acquisition. Calculation principles of 3D coordinates from the real-time data of all the involved sensors are discussed. The field tests were carried out in a controlled environment of a parking lot and at different velocities. Experiments were carried out to test the ability of the GNSS/INS system to cope with difficult conditions, e.g. sudden movements due to cornering or swerving. The accuracy of the resulting MLS point cloud is evaluated with respect to high-accuracy static terrestrial laser scanning data. Problems regarding combining LIDAR, GNSS and INS sensors are outlined, as well as the initial accuracy assessments. Initial tests revealed errors related to insufficient quality of inertial data and a need for the trajectory post-processing calculations. Although this study was carried out while the system was mounted on a car, there is potential for operating the system on an unmanned aerial vehicle, all-terrain vehicle or in a backpack mode due to its relatively compact size.
\end{abstract}

\section{INTRODUCTION}

MLS systems are used to gather 3D spatial data (i.e. point cloud) on the move. The main components of MLS systems are a LIDAR scanner, a GNSS (Global Navigation Satellite System) receiver and an INS (Inertial Navigation System), which are synchronized and placed on a moving platform. Odometer data and dual GNSS antennas for determining the heading are often implemented to improve the quality of the result. The scientific research of MLS, of which there is a considerable amount, has mostly dealt with calibrating and data processing, mainly the automatic extraction of features from a point cloud.

The point cloud calculation and direct georeferencing of MLS data share the same principles as Airborne Laser Scanning (ALS), see e.g. Wehr and Lohr (1999). In feature (such as pavements, technical utilities, terrain relief, facades) extraction most of the same principles apply as with terrestrial laser scanning (TLS). Such examples include extraction of building features by $\mathrm{Pu}$ et al. (2006), extraction of a tunnel liner by Yoon et al. (2009) and reconstructing tree crowns by Pyysalo et al. (2002). For bare-earth extraction algorithms and their performance analysis see Sithole and Vosselman (2004), Julge et al. (2014a) and references therein. Recognizing and extracting features from MLS point clouds has been discussed by e.g. Pu et al. (2011), Yang et al (2013) and Guan et al. (2014).

* Corresponding author
Some examples of previous research of MLS include also a review of mobile mapping and surveying technologies (Puente et al., 2013), geometric validation of ground-based MLS systems (Barber et al., 2008), creating and testing multiplatform MLS systems (Kukko et al., 2012), evaluating MLS systems for tree measurements (Jaakkola et al., 2010) and environment mapping (Jaakkola, 2015).

MLS has developed rapidly in the recent years and there are several commercial products available. Companies that provide commercial MLS systems include Riegl, Trimble, 3D Laser Mapping, TopScan, Dynascan, Optech etc. However, complete commercial MLS systems are usually expensive. Purchasing the required sensors separately is more cost-effective but does require time-consuming work to integrate the systems and calculate the final point clouds. However, there is a possibility to adapt and fine-tune the system according to the specifics of the task at hand. Complete hardware and software solutions utilizing Velodyne scanners, either VLP-16 or larger HDL-32E model, include Routescene LidarPod, Topcon IP-S3, Phoenix Aerial Systems, Hypack, etc.

Accordingly, this paper describes an in-house assembled compact and relatively low-cost MLS system consisting of a Velodyne VLP-16 LIDAR and Advanced Navigation Spatial Dual GNSS/INS system, as well as related data processing and the accuracy assessment of the initial results. The MLS data are compared with high resolution and high accuracy (sub-cm) TLS reference data. The ultimate goal is to use MLS point clouds in 
conjunction with photos from a $360^{\circ}$ spherical camera Ladybug5 to provide a possibility for reliable measurements in the Street-U street view application. However, this is not yet implemented.

The outline of the paper is as follows. First the specifications of the used sensors and the basic principles of point cloud calculation are reviewed, after which the case study at hand is described including the methodology. Then the achieved results and initial accuracy assessment are presented. Lastly, a brief summary and a description of goals for further research concludes the paper.

\section{SYSTEM DESCRIPTION}

\subsection{Sensors}

The assembled MLS system is based on a Velodyne VLP-16 compact LIDAR sensor and a dual antenna GNSS/INS system Advanced Navigation Spatial Dual. The specifications of the used sensors are outlined in Table 1. The two systems are synchronized with one another by sending a pulse-per-second (PPS) signal in conjunction with a once-per-second NMEA \$GPRMC sentence from the GNSS/INS system to the LIDAR. The Spatial Dual system is connected to a data logging computer with RS-232 cables. The data packets of VLP-16 are output through an Ethernet port.

\subsection{Point cloud calculation}

The point cloud coordinates are calculated by combining distance and horizontal/vertical angle data from the LIDAR sensor and the position and inertial data from the GNSS/INS system.

The point cloud calculation method (specific to Velodyne scanners) is as follows. The data packets received through an Ethernet port are parsed for rotational angles, measured range to the object, calibrated reflectivities and time stamp. The VLP-16 reports spherical coordinates $(R, \omega, \alpha)$. Therefore, a transformation is needed to convert to $x, y, z$ coordinates (Fig. 1). The vertical/elevation angle $(\omega)$ is fixed and is determined by the Laser ID, which is indicated by the position of the return in the data packet. The horizontal angle/azimuth $(\alpha)$ is reported at the beginning of every other firing sequence, and the distance is reported in the two distance bytes. Points within one-meter distance are ignored. (VLP-16 user manual)

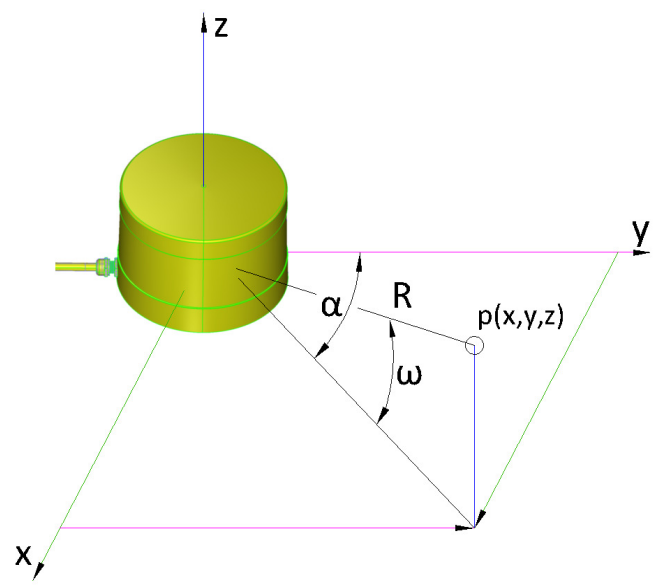

Figure 1. The interrelations between spherical polar coordinates of VLP-16 to $x, y, z$ coordinates.

\begin{tabular}{|c|c|}
\hline \multicolumn{2}{|c|}{ Velodyne VLP-16 LIDAR } \\
\hline Sensor & $\begin{array}{l}\text { Time of flight distance measurement } \\
16 \text { channels } \\
\text { Range up to } 100 \mathrm{~m} \\
\text { Accuracy: } \pm 3 \mathrm{~cm} \\
\text { Vertical field of view: } 30^{\circ} \\
\text { Horizontal field of view: } 360^{\circ} \\
\text { Dual returns } \\
\text { Vertical angular resolution: } 2^{\circ} \\
\text { Horizontal angular resolution: } 0.1^{\circ} \ldots 0.4^{\circ} \\
\text { Rotation rate: } 5 \ldots 20 \mathrm{~Hz}\end{array}$ \\
\hline Laser & $\begin{array}{l}\text { Class } 1 \text { - eye safe } \\
\text { Wavelength }-903 \mathrm{~nm} \\
\end{array}$ \\
\hline Mechanical & $\begin{array}{l}\text { Weight: } 830 \text { grams (without cabling) } \\
\text { Dimensions: } 103 \mathrm{~mm} \text { diameter } \mathrm{x} 72 \mathrm{~mm} \text { height }\end{array}$ \\
\hline Electrical & $\begin{array}{l}\text { Power consumption: } 8 \mathrm{~W} \\
\text { Operating voltage: } 9 \text { - } 32 \mathrm{~V} \mathrm{DC}\end{array}$ \\
\hline Output & $\begin{array}{l}\text { Up to } 300000 \text { points/second } \\
100 \text { Mbps Ethernet connection } \\
\text { UDP packets containing } \\
\text {-Distances } \\
\text {-Calibrated reflectivities } \\
\text {-Rotation angles } \\
\text {-Synchronized time stamps ( } \mu \text { s resolution) } \\
\text { \$GPRMC NMEA sentence from GPS receiver }\end{array}$ \\
\hline \multicolumn{2}{|c|}{ Advanced Navigation Spatial Dual GNSS/INS system } \\
\hline Navigation & $\begin{array}{l}\text { Horizontal accuracy: } 1.2 \mathrm{~m} \\
\text { Vertical accuracy: } 2.0 \mathrm{~m} \\
\text { Horizontal accuracy (RTK): } 0.008 \mathrm{~m} \\
\text { Vertical accuracy (RTK): } 0.015 \mathrm{~m} \\
\text { Roll and Pitch dynamic accuracy: } 0.15^{\circ} \\
\text { Heading dynamic accuracy: } 0.1^{\circ} \\
\text { Velocity accuracy: } 0.007 \mathrm{~m} / \mathrm{s}\end{array}$ \\
\hline GNSS & $\begin{array}{l}\text { Model: Trimble BD982 } \\
\text { Antennas: } 2 \text { x Antcom G5Ant-53A4T1 } \\
\text { GPS L1, L2, L5 } \\
\text { GLONASS L1, L2 } \\
\text { GALILEO E1, E5 } \\
\text { BeiDou B1, B2 } \\
\text { Update rate: } 20 \mathrm{~Hz}\end{array}$ \\
\hline Mechanical & $\begin{array}{l}\text { Weight: } 285 \text { grams (without cabling and GNSS } \\
\text { antennas) } \\
\text { Dimensions: } 90 \times 127 \times 31 \mathrm{~mm}\end{array}$ \\
\hline Electrical & $\begin{array}{l}\text { Power consumption: } 220 \mathrm{~mA} \text { at } 12 \mathrm{~V} \\
\text { Operating voltage: } 9-36 \mathrm{~V} \text { DC } \\
\end{array}$ \\
\hline Output & $\begin{array}{l}\text { Interface: RS232 } \\
\text { Baudrate: } 4800 \ldots 1000000 \\
\text { Protocol: AN Packet Protocol or NMEA }\end{array}$ \\
\hline
\end{tabular}

Table 1. Velodyne VLP-16 and Advanced Navigation Spatial Dual specifications (VLP-16 datasheet, Spatial Dual datasheet)

The coordinates $x, y, z$ with respect to the VLP-16 centre can be calculated by:

$$
\left[\begin{array}{l}
x \\
y \\
z
\end{array}\right]=\left[\begin{array}{c}
R \cdot \cos \omega \cdot \sin \alpha \\
R \cdot \cos \omega \cdot \cos \alpha \\
R \cdot \sin \omega
\end{array}\right]
$$

where $\quad x, y, z=$ coordinates of an individual survey point $R=$ range from scanner to survey point

$\omega=$ vertical angle

$\alpha=$ horizontal angle 
These calculated 3D coordinates are in a local (sensor's) frame. In order to georeference the point cloud and display consecutive point clouds together as a unified point cloud, GNSS and INS data need to be taken into account. This is referred to as direct georeferencing, which is defined as a transformation between the sensor coordinates frame and the geodetic reference frame. This has been previously described by e.g. Schwarz et al. (1993) Cramer et al. (2000), Grejner-Brzezinska (1999) and Baltsavias (1999). Although these articles concentrate on direct georeferencing of photogrammetry and airborne laser scanning data, the same basic principles apply here as well.

The custom software for point cloud computations was written in Java programming language. The inputs are LIDAR data packets and a text file containing GNSS/INS data. The software checks the time-stamps of both datasets and calculates geodetic coordinates based on the rotational angles and range from the LIDAR, the position and heading data from the GNSS and the roll and pitch data from the INS. Output is a point cloud file in ASCII text format containing 3D coordinates and reflective intensity of points.

\section{METHODOLOGY}

\subsection{Field work and study area}

The initial MLS field measurements were done in December of 2015. The sensors were placed on a sturdy wood plate on the roof rack of a car. The LIDAR was positioned in the back of the car looking down at a $30^{\circ}$ angle. The inertial measurement unit (IMU) was located in the centre and the dual GNSS antennas were located on either side of it, see Fig. 2. Real time kinematic GNSS and INS data were recorded, synchronously with the VLP-16 data packets.

The study area was a 50x100m parking lot of Tallinn University of Technology. The main objects of interest were the asphaltsurface of the parking lot and the façade of an adjacent building, see Fig. 2. Data was collected while moving straight in a steady speed, cornering, swerving and alternating between braking and accelerating.

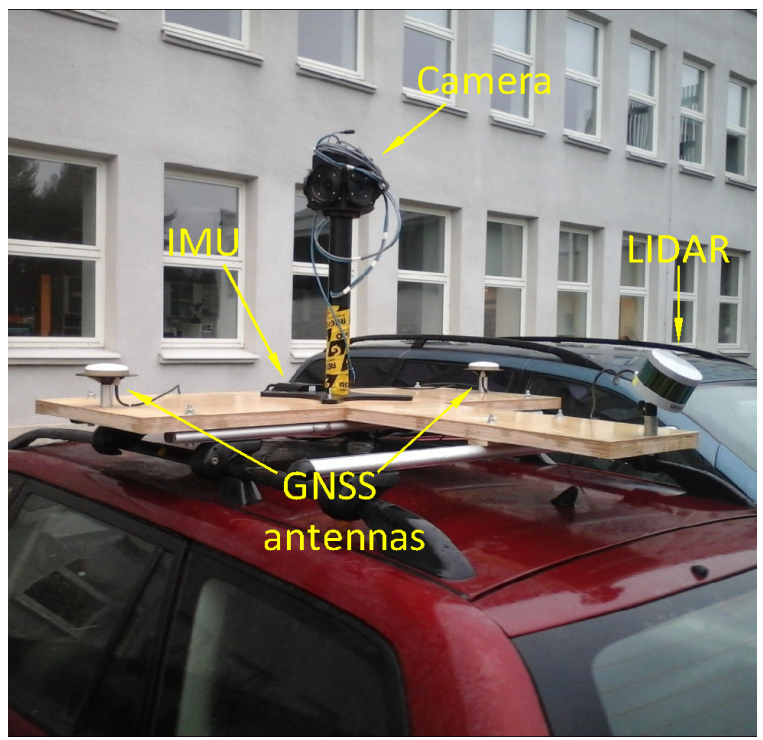

Figure 2. The placement of the VLP-16 and Spatial Dual sensors on the roof of a vehicle. $360^{\circ}$ spherical camera Ladybug 5 can be seen in the centre. The surveyed building façade in the background.

\subsection{Reference data}

The reference data was collected with a time-of-flight terrestrial laser scanner Leica ScanStation C10 with an average spatial resolution of $\sim 2500$ points $/ \mathrm{m}^{2}(2 \times 2 \mathrm{~cm})$. The scanner is rated for $6 \mathrm{~mm}$ positional accuracy. The point cloud registration and georeferencing of the TLS data were conducted based on 3 reference points which were coordinated with survey-grade GNSS measurements. The scanning was performed from three scan positions in order to sufficiently cover the asphalt-surface of the parking lot and the façade of the adjacent building. The TLS point cloud was clipped to only the area of interest and the measurement noise, as well as points reflected from obstructions (e.g. cars) were removed.

\section{RESULTS AND ACCURACY ASSESSMENT}

The resulting MLS point cloud (Fig. 3) was calculated with the method discussed in section 2.2. Overall more than 10 million points were measured during the MLS test survey.

The final MLS point cloud was compared with TLS data. The datasets indicated reasonable agreement near the trajectory of the vehicle. However, the errors became linearly larger when moving further away from the trajectory. The errors are mostly attributed to insufficient quality of the INS data, especially the roll misalignment. This is more evident during cornering and swerving. It should also be noted, that the accuracy of the distance measured with VLP-16 is $\pm 3 \mathrm{~cm}$ (as shown in Table 1), which is considerably less accurate than that of TLS (see Section 3.2).

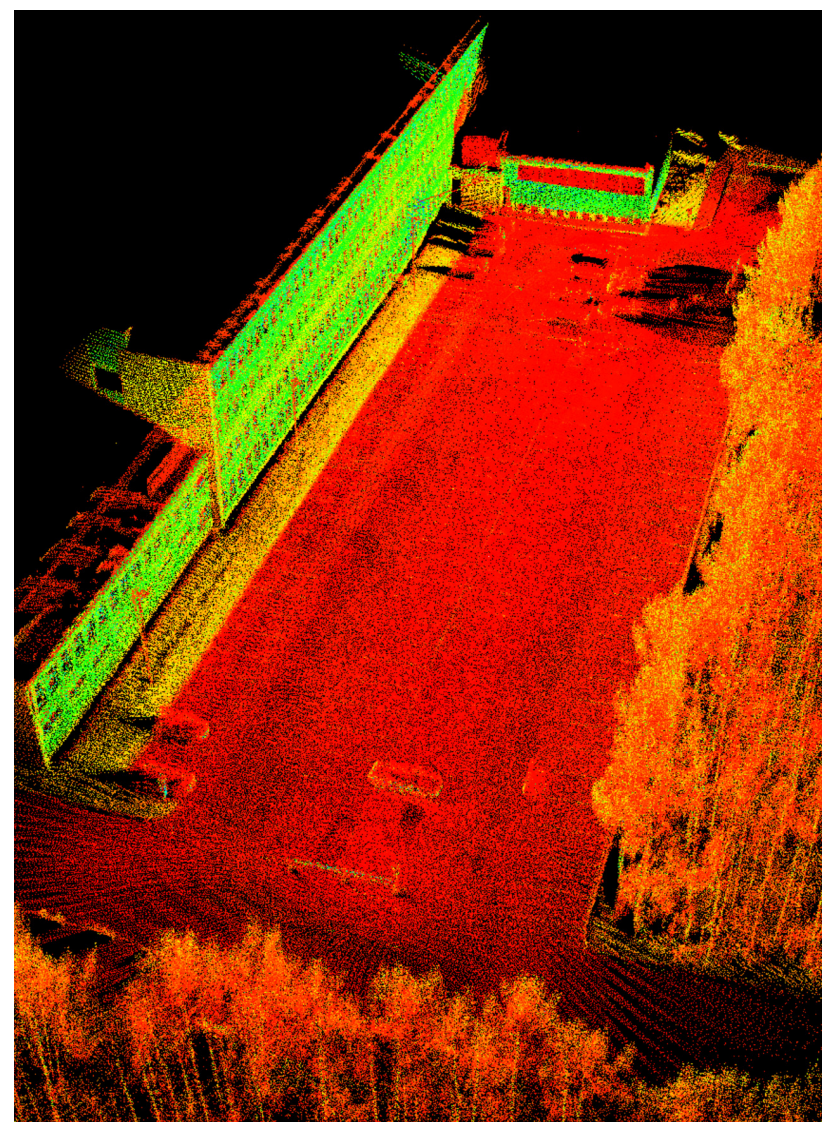

Figure 3. MLS point cloud of the study area. Colours show the calibrated reflectivities of surveyed points. 


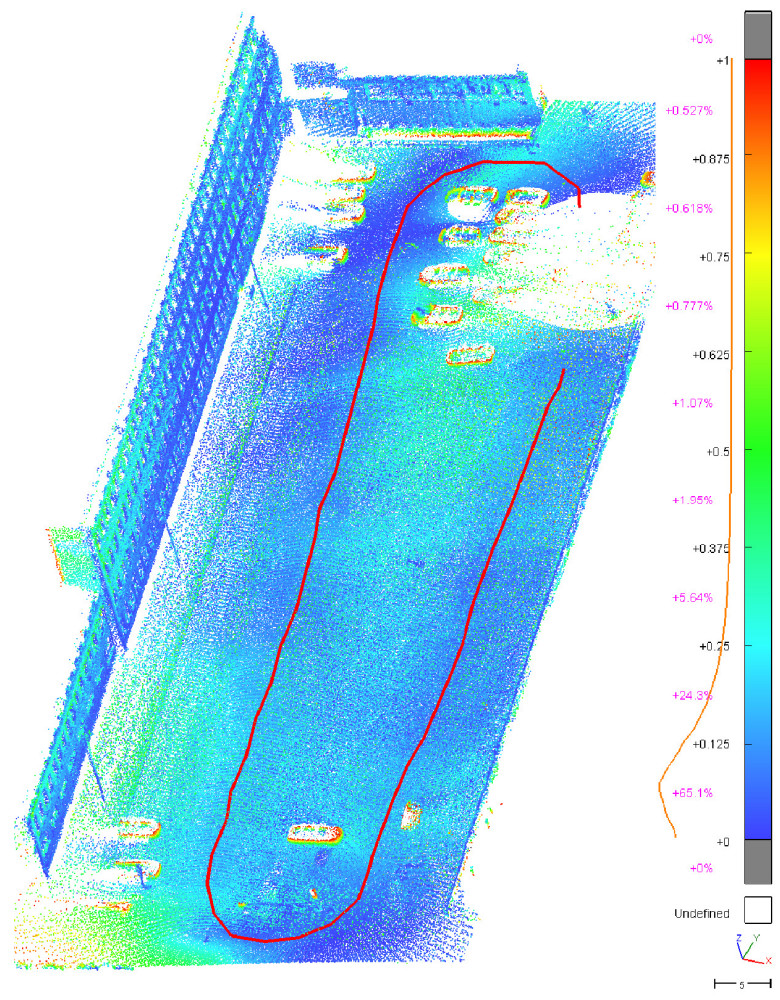

Figure 4. 3D discrepancies between TLS and MLS data (the absolute values of differences) in the test site. The red line denotes the clockwise trajectory of the vehicle. The empty data pockets are attributed to the cars in the parking lot. The horizontal scale denotes 5 metres.

The 3D discrepancies between the MLS and TLS clouds are shown in Fig. 4. The absolute values of the discrepancies mostly did not exceed $15 \mathrm{~cm}$ (blue points in Fig.4) with the root mean square error (RMSE) being $7.5 \mathrm{~cm}$. The differences on the façade were mostly less than $10 \mathrm{~cm}$ but there is evidence of the two datasets being slightly tilted with respect to one another both horizontally and vertically. The first is presumably caused by the accuracy of position, i.e. GNSS data, and the second by roll misalignment. However, the dimensions (both height and width) of the building derived from TLS and MLS data indicated good agreement, within $1 \mathrm{~cm}$ vertically and within 3 cm horizontally.

According to the histogram (on the right hand side of Fig. 4), $65 \%$ of the detected discrepancies were within $12.5 \mathrm{~cm}$ and $90 \%$ of discrepancies within $25 \mathrm{~cm}$. However, since the MLS data seem to be most affected by the roll misalignment error, then larger errors are observed with points measured while cornering and more than 5 metres away from the trajectory. Therefore, the isolated parts of the point cloud were evaluated separately.

On Fig. 5, vertical discrepancies between the TLS data and a segment of the MLS point cloud measured during cornering is shown. The differences increase during the cornering. At first, the roll is under-compensated (indicated by the higher (red) points on the "inside" corner at the top left of Fig. 5 which were measured at the start of the cornering manoeuvre) but later, the results show over-compensation (indicated by the higher (red) points on the "outside" corner at the bottom left of Fig. 5, which were measured in the end of the cornering manoeuvre).

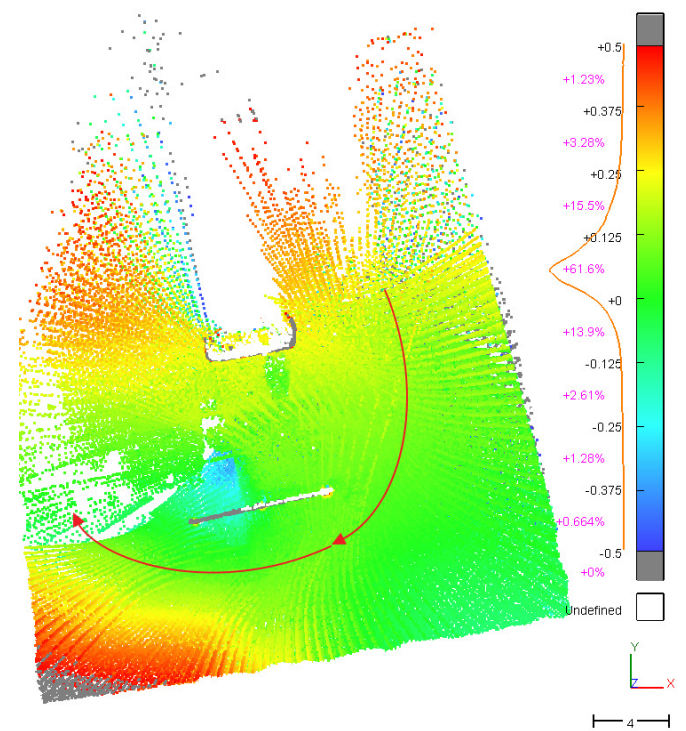

Figure 5. Vertical discrepancies between the TLS and MLS data acquired during cornering. The red arrowed line denotes the clockwise trajectory of the vehicle.

In the latter case the (red) points in the "outside corner" appear to be linearly higher than TLS data and vice versa, even though the roll of the vehicle in corners would cause the points in the "outside corner" to be lower if roll was not taken into account at all. Near the trajectory of the vehicle the discrepancies are within $10 \mathrm{~cm}$ (green points). It is worth noting that the density of points is higher near the trajectory and therefore $75 \%$ of the discrepancies are within $12.5 \mathrm{~cm}$ but larger than $50 \mathrm{~cm}$ differences occur further away from the trajectory.

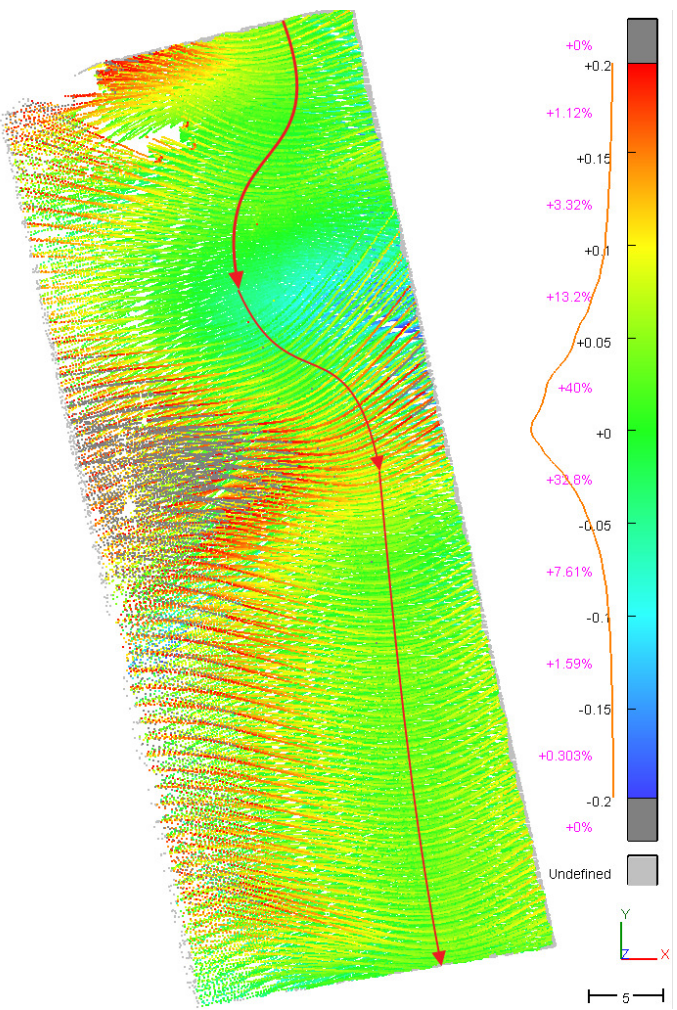

Figure 6. Vertical discrepancies between the TLS and MLS data gathered during a swerving manoeuvre. The red arrowed line denotes the trajectory of the vehicle. 
The same effect can be observed by examining the results collected during a swerving manoeuvre (Fig. 6). At first the roll is under-compensated but at the end it is over-compensated. Although there are large errors further away from the trajectory, the accuracy of the points measured near the trajectory is within a couple of $\mathrm{cm}$. Overall, ca $75 \%$ of the discrepancies do not exceed $\pm 5 \mathrm{~cm}$.

\section{CONCLUSION AND FUTURE RESEARCH}

This paper presented the description of a relatively compact and low-cost MLS system, as well as the results and accuracy assessment of the initial case study. The initial tests indicated quite large errors mostly due to poor rotational angle data from the INS, especially roll misalignment.

The achieved results may be acceptable in some cases where high accuracy is not necessary, e.g. using single profiles to estimate the condition and smoothness of a road surface or estimating volumes of land mass transportations. For example, such MLS can also be used for quantifying the erosion and sedimentation over long portions of sand beaches. Our previous studies (Julge et al., 2014b, Eelsalu et al., 2015) have exploited both TLS and ALS data for this purpose. However, TLS survey is very time- and labour-consuming, whereas ALS data (acquired during national mapping campaigns) are not always available nor up-to-date. Even the current state of the assembled MLS system would provide a faster alternative with sufficient accuracy.

In order to make the results usable in other areas, further research and development is needed. Further experiments will include recording raw GNSS and INS data and using that information in post-processing trajectory calculations. Also there is a space for further calibration and improvements in the quality of inertial data. This will hopefully result in more accurate $3 \mathrm{D}$ point clouds.

Another direction for further research is to implement an algorithm which checks the distance of measured points from the scanner in overlapping areas and removes points that have been measured from adjacent/parallel MLS routes. These measurements are more susceptible to errors caused by poor inertial data and therefore less accurate. Also feature detection (mainly curb stones, posts and building outlines) will be integrated into the point cloud calculation software.

One of the main objectives is to use the MLS point clouds in conjunction with the image data from Ladybug5 camera (shown in Fig. 2) in order to add a possibility for reliable measurements in the existing Street-U street view online application (currently similar to Google Street View).

After the successful implementation of the MLS system on a car, other vehicles for mounting the system will be considered, e.g. an unmanned aerial vehicle, all-terrain vehicle or a "backpack".

\section{ACKNOWLEDGEMENTS}

This research has been supported by European Union Regional Development Fund. The used Velodyne VLP-16 LIDAR and Leica ScanStation C10 were purchased within frames of Estonian scientific equipment purchase support programme (project SF0140007s11AP14) and Estonian Research Infrastructures Roadmap object Estonian Environmental
Observatory (project No. AR12019). The participation in the XXIII ISPRS Congress in Prague is (partially) funded with SA Archimedes' Kristjan Jaak Scholarship.

\section{REFERENCES}

"Advanced Navigation Spatial Dual datasheet" http:// www.advancednavigation.com.au/sites/advancednavigation.com .au/files/Spatial\%20Dual\%20Datasheet.pdf (15 Feb. 2016)

Baltsavias, E.P., 1999. Airborne laser scanning: basic relations and formulas. ISPRS Journal of Photogrammetry and Remote Sensing, 54(2), pp. 199-214.

Barber, D., Mills, J. and Smith-Voysey, S., 2008. Geometric validation of a ground-based mobile laser scanning system. ISPRS Journal of Photogrammetry and Remote Sensing, 63(1), pp. 128-141.

Cramer, M., Stallmann, D. and Haala, N., 2000. Direct georeferencing using GPS/inertial exterior orientations for photogrammetric applications. In: International Archives of Photogrammetry and Remote Sensing, 33(B3/1; PART 3), pp. 198-205.

Eelsalu, M., Soomere, T. and Julge, K., 2015. Quantification of changes in the beach volume by the application of an inverse of the Bruun Rule and laser scanning technology. Proceedings of the Estonian Academy of Sciences, 64(3), pp. 240-248.

Guan, H., Li, J., Yu, Y., Wang, C., Chapman, M. and Yang, B., 2014. Using mobile laser scanning data for automated extraction of road markings. ISPRS Journal of Photogrammetry and Remote Sensing, 87, pp. 93-107.

Grejner-Brzezinska, D.A., 1999. Direct exterior orientation of airborne imagery with GPS/INS system: Performance analysis. Navigation, 46(4), pp. 261-270.

Jaakkola, A., 2015. Low-cost Mobile Laser Scanning and its Feasibility for Environmental Mapping. (PhD thesis)

Jaakkola, A., Hyyppä, J., Kukko, A., Yu, X., Kaartinen, H., Lehtomäki, M. and Lin, Y., 2010. A low-cost multi-sensoral mobile mapping system and its feasibility for tree measurements. ISPRS Journal of Photogrammetry and Remote Sensing, 65(6), pp. 514-522.

Julge, K., Ellmann, A. and Gruno, A., 2014a. Performance analysis of freeware filtering algorithms for determining ground surface from airborne laser scanning data. Journal of Applied Remote Sensing, 8(1), pp. 083573-083573.

Julge, K., Eelsalu, M., Grunthal, E., Talvik, S., Ellmann, A., Soomere, T. and Tõnisson, H., 2014b. Combining airborne and terrestrial laser scanning to monitor coastal processes. In: Baltic International Symposium (BALTIC), 2014 IEEE/OES pp. 1-10.

Kukko, A., Kaartinen, H., Hyyppä, J. and Chen, Y., 2012. Multiplatform mobile laser scanning: Usability and performance. Sensors, 12(9), pp. 11712-11733.

Pu, S., Rutzinger, M., Vosselman, G. and Elberink, S.O., 2011. Recognizing basic structures from mobile laser scanning data for road inventory studies. ISPRS Journal of Photogrammetry and Remote Sensing, 66(6), pp. S28-S39. 
$\mathrm{Pu}$, S. and Vosselman, G., 2006. Automatic extraction of building features from terrestrial laser scanning. International Archives of Photogrammetry, Remote Sensing and Spatial Information Sciences, 36(5), pp. 25-27.

Puente, I., González-Jorge, H., Martínez-Sánchez, J. and Arias, P., 2013. Review of mobile mapping and surveying technologies. Measurement, 46(7), pp. 2127-2145.

Pyysalo, U. and Hyyppa, H., 2002. Reconstructing tree crowns from laser scanner data for feature extraction. International Archives Of Photogrammetry Remote Sensing And Spatial Information Sciences, 34(3/B), pp. 218-221.

Schwarz, K.P., Chapman, M.A., Cannon, M.W. and Gong, P., 1993. An integrated INS/GPS approach to the georeferencing of remotely sensed data. Photogrammetric engineering and remote sensing, 59(11), pp. 1667-1674.

Sithole, G. and Vosselman, G., 2004. Experimental comparison of filter algorithms for bare-Earth extraction from airborne laser scanning point clouds. ISPRS journal of photogrammetry and remote sensing, 59(1), pp. 85-101.

"Velodyne VLP-16 datasheet" http://velodynelidar.com/ docs/datasheet/63-9229_Rev-C_VLP16_Datasheet_Web.pdf

(19 Feb. 2016)

"Velodyne VLP-16 User's manual and programming guide" http://velodynelidar.com/docs/manuals/63-9243\%20Rev\%20B $\% 20$ User\%20Manual\%20and\%20Programming\%20Guide,VLP -16.pdf (11 Feb. 2016)

Wehr, A. and Lohr, U., 1999. Airborne laser scanning-an introduction and overview. ISPRS Journal of Photogrammetry and Remote Sensing, 54(2), pp. 68-82.

Yang, B., Fang, L. and Li, J., 2013. Semi-automated extraction and delineation of $3 \mathrm{D}$ roads of street scene from mobile laser scanning point clouds. ISPRS Journal of Photogrammetry and Remote Sensing, 79, pp. 80-93.

Yoon, J.S., Sagong, M., Lee, J.S. and Lee, K.S., 2009. Feature extraction of a concrete tunnel liner from 3D laser scanning data. NDT \& E International, 42(2), pp. 97-105. 